
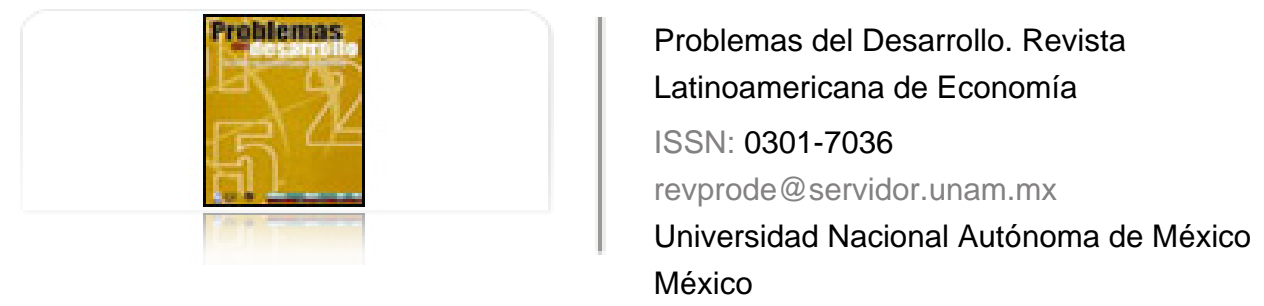

Morales Sánchez, Mario Alberto

TEORÍA ECONÓMICA EVOLUTIVA DE LA EMPRESA: ¿UNA ALTERNATIVA A LA TEORÍA NEOCLÁSICA?

Problemas del Desarrollo. Revista Latinoamericana de Economía, vol. 40, núm. 158, julio-septiembre, 2009, pp. 161-183

Universidad Nacional Autónoma de México

Distrito Federal, México

Disponible en: http://www.redalyc.org/articulo.oa?id=11820200007

Cómo citar el artículo

- Número completo

- Más información del artículo

Página de la revista en redalyc.org

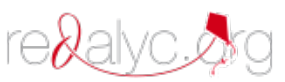

Sistema de Información Científica

Red de Revistas Científicas de América Latina, el Caribe, España y Portugal Proyecto académico sin fines de lucro, desarrollado bajo la iniciativa de acceso abierto 


\title{
TEORÍA ECONÓMICA EVOLUTIVA DE LA EMPRESA: ¿UNA ALTERNATIVA A LA TEORÍA NEOCLÁSICA?
}

\author{
Mario Alberto Morales Sánchez*
}

Fecha de recepción: 17 de abril de 2009. Fecha de aceptación: 31 de julio de 2009.

\section{Resumen}

Este trabajo presenta una reflexión crítica sobre las aportaciones conceptuales de la teoría económica evolutiva de la empresa. Sus contribuciones se comparan con el limitado alcance de la teoría neoclásica al explicar los factores internos de desempeño de la empresa, ya que la consideran una función técnica de producción. Al mismo tiempo, el artículo se ocupa especialmente de exponer los puntos principales que definen la propuesta evolutiva de la empresa, discutiendo el progreso conceptual y explicativo de sus postulados y sus limitaciones intrínsecas. Se concluye con un balance conceptual entre los principales méritos de la teoría económica evolutiva de la empresa y los puntos débiles que exhibe esta corriente teórica.

Palabras clave: empresa, teoría evolutiva, conocimiento, agente, rutina.

\begin{abstract}
This study reflects critically on the conceptual contributions made by the evolutionary theory of the firm. Its contributions are compared with the limited scope of neoclassical theory to explain the internal factors of company development, as they involve a technical production function. At the same time, the article is especially concerned to clarify the main points that define the company's evolutionary purpose, discussing the conceptual and explicative progress of its postulates and its intrinsic limitations. It concludes with a conceptual balance of the main merits of the evolutionary theory of the firm and the weak points exhibited in this theoretical current.
\end{abstract}

Keywords: company, evolutionary theory, knowledge, agent, routine.

* Maestro en Economía y Gestión de la Innovación UAM-X. Profesor adjunto del Posgrado de la Facultad de Economía UNAM. Correo electrónico: alberto_rork@ yahoo.com 
Résumé

Ce travail présente une réflexion critique sur les apports conceptuels de la théorie économique évolutive de l'entreprise. Ses contributions sont comparées à la portée limitée de la théorie néoclassique pour expliquer les facteurs internes de performance de l'entreprise, du fait de l'envisager comme une fonction technique de production. En même temps, l'article s'attache tout particulièrement à exposer les points principaux qui définissent la proposition évolutive de l'entreprise, discutant le progrès conceptuel et explicatif de ses postulats et ses limitations intrinsèques. Il est conclu par un bilan conceptuel entre les principaux mérites de la théorie économique évolutive de l'entreprise et les points faibles dont fait preuve ce courant théorique.

Mots clés: entreprise, théorie évolutive, connaissance, agent, routine.

\section{Resumo}

Este trabalho apresenta uma reflexão crítica sobre as aportações conceituais da teoria econômica evolutiva da empresa. Suas contribuições se comparam com o limitado alcance da teoria neoclássica ao explicar os fatores internos de desempenho da empresa, uma vez que a consideram uma função técnica de produção. Ao mesmo tempo, o artigo se ocupa especialmente de expor os pontos principais que definem a proposta evolutiva da empresa, discutindo o progresso conceitual e explicativo de seus postulados e suas limitações intrínsecas. Conclui-se com um balanço conceitual entre os principais méritos da teoria econômica evolutiva da empresa e as debilidades que apresenta esta corrente teórica.

Palavras-chave: empresa, teoria evolutiva, conhecimento, agente, rotina. 


\section{Introducción}

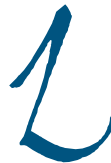

a exposición general de los fundamentos epistemológicos de la teoría de la empresa desde la perspectiva de la teoría económica evolutiva (TEE) pretende situar el concepto de empresa propuesto por la TEE en una discusión actual para evaluar sus aportaciones teóricas, sus límites y perspectivas.

Resulta pertinente considerar que aunque este trabajo se concentra en las contribuciones de la TEE, esta corriente forma parte de un conjunto amplio de teorías que han intentado explicar la naturaleza de la empresa desde una perspectiva económica. Dichas teorías se extienden desde las aportaciones clásicas de Smith y Marx referentes a la organización interna basada en la división del trabajo y la cooperación, hasta teorías más recientes, entre las que destaca la propuesta de F. Knigth respecto de los mecanismos internos de organización como una forma de afrontar la incertidumbre que caracteriza al ambiente, o la de F. Hayek, quien destaca la importancia del conocimiento como un insumo productivo (en relación con estos autores, véase Putterman y Kroszner, 2006).

En la actualidad, el debate se enriquece con otras vertientes contemporáneas. Por mencionar sólo algunas, conviene remitirse al trabajo de Chandler (1977), quien destaca la importancia de la jerarquía empresarial como medio de reemplazar al mercado en la distribución interna de recursos; o la teoría de la empresa basada en los recursos, que se desprende del trabajo clásico de E. Penrose (1959) y cuya ramificación más destacada en la actualidad corresponde con la teoría de las competencias centrales (Prahalad y Hamel, 1990) y competencias tecnológicas (Patel y Pavitt, 1994). Para el debate actual también resultan relevantes las aportaciones del institucionalismo clásico, representado por autores como Coase (1937) y Williamson (1989), quienes plantean que la jerarquía es una alternativa al mercado ya que evita costos de transacción ${ }^{1}$.

Lo que se denomina TEE es la corriente de pensamiento que se consolida a partir del trabajo seminal de Nelson y Winter (1982), An Evolutionary Theory of Economic Change, que introduce nuevamente en la discusión económica la necesidad de explicar el desempeño de la empresa a partir de los principios de la teoría evolutiva² ${ }^{2}$ El

1 La construcción de una teoría alternativa de la empresa consistente, que dé cuenta de su naturaleza ontológica y su constitución interna, requiere la revisión crítica de estas aportaciones con la finalidad de rescatar y articular los elementos más importantes que las componen. Esta perspectiva coincide con la opinión de autores como Valdaliso y López (2000), quienes destacan el valor de una reconstrucción sintética de diversas teorías de la empresa.

2 Quizá el primer economista destacado en intentarlo explícitamente fue Schumpeter, al proponer que el análisis de los procesos económicos debe centrarse en su dinámica. Y afirma: "el punto esencial que hay que tener en cuenta consiste en que, al tratar el capitalismo, nos 
programa de la TEE incluye un conjunto vasto de temas de investigación que van desde los fundamentos microeconómicos evolutivos, hasta el análisis competitivo de los sistemas agregados y de la dinámica económica general. Como base para la discusión sólo se considerará del trabajo de Nelson y Winter la parte que se refiere a los elementos ontológicos de definición de la empresa; no sólo por considerar que es la explicación más coherente formulada hasta ahora por la perspectiva evolutiva, sino porque como plantean Felin y Foss (2004: 11), desde que el término rutina organizacional fue retomado por Nelson y Winter, ha sufrido muy poco avance explicativo. Se parte de la hipótesis de que los postulados establecidos por Nelson y Winter representan el máximo avance conceptual y explicativo en cuanto a la definición evolutiva de empresa $a^{3}$.

Del trabajo de Nelson y Winter (1982) puede inferirse que uno de los principales propósitos en la microfundamentación de la TEE es construir una teoría de la empresa que cumpla con dos funciones epistemológicas centrales: la primera, identificar de manera crítica las insuficiencias explicativas de la teoría ortodoxa ${ }^{4}$ respecto a la funcionalidad interna de la unidad básica de producción capitalista; la segunda, postular una alternativa teórica que explique el comportamiento de los sistemas económicos agregados. En su aspecto microanalítico, dicho programa pretende ser tanto una síntesis de teorías previas como una crítica a las concepciones tradicionales de la empresa en la teoría neoclásica.

La exposición de este trabajo comienza con el planteamiento crítico que hace la TEE de la teoría neoclásica; posteriormente se identifican de forma crítica los temas centrales de su programa de investigación. Dos interrogantes guían esta exposición: ¿En qué radica el progreso conceptual y explicativo de la TEE en su definición de empresa? ¿Cuáles son las ideas centrales que identifican su programa de investigación? Asimismo, se evaluará la consistencia conceptual de algunas de sus ideas centrales.

enfrentamos con un proceso evolutivo (...) El capitalismo es, por naturaleza, una forma o método de transformación económica y no solamente no es jamás estacionario, sino que no puede serlo nunca" (Schumpeter [1942] 1983: 120).

3 Los conceptos progreso conceptual y progreso explicativo se entienden en el sentido de Kitcher (2001). Existirá progreso conceptual cuando las categorías de significación utilizadas para explicar los fenómenos puedan ajustarse de mejor manera a su naturaleza y a sus relaciones intrínsecas, y cuando sea posible establecer mejores especificaciones con respecto a los referentes actuales. Por otra parte, habrá progreso explicativo cuando sea posible mejorar la concepción que se tiene de las relaciones causales determinantes del comportamiento de los fenómenos, es decir, acerca de la estructura concreta de la naturaleza.

4 Nelson y Winter (1982) entienden por teoría ortodoxa todos aquellos postulados estándar en los que se sustentan la mayor parte de los manuales tradicionales de economía microeconómica neoclásica y sus consecuentes derivaciones macroeconómicas, los cuales explican el comportamiento de la empresa en función de la determinación de precios y cantidades de equilibrio en un contexto específico determinado por ciertas condiciones de mercado. 


\section{Crítica a la teoría neoclásica de la empresa desde la perspectiva evolutiva}

La teoría evolutiva de la empresa intenta erigirse como una alternativa crítica focalizada principalmente en algunos aspectos de la microeconomía neoclásica ${ }^{5}$. El primero se refiere a que la empresa no puede ser representada sólo, o primordialmente, como una función de producción; es pertinente "expandir" el análisis económico hacia los procesos técnicos y organizacionales que ocurren en su interior. Por otra parte, establece la imposibilidad de que las empresas tengan como único objetivo el maximizar sus beneficios a través de una conducta perfectamente racional, principalmente por la incertidumbre del ambiente. Por último, afirma que las empresas no se desenvuelven como actores pasivos en su entorno (precio-aceptantes); sus decisiones son capaces de alterar los mercados mediante la modificación en la calidad de los bienes que producen.

A pesar de que el objetivo primordial de la TEE se dirige a la explicación dinámica de "los sistemas agregados (larger systems) y no hacia los actores individuales", las exigencias epistemológicas que reivindica implican el desarrollo de una microfundamentación del comportamiento empresarial, basada en una "teoría plausible de las capacidades y comportamiento de la empresa que sea consistente con la evidencia microeconómica" (Nelson y Winter, 1982: 51-52). Dicha teoría se construye en un primer momento a través de la crítica, la cual se concentra fundamentalmente en la incapacidad de la teoría ortodoxa para explicar los procesos que se desarrollan al interior de la empresa, los cuales corresponden tanto a la producción de objetos útiles (tangibles o intangibles) como a la dimensión organizacional. Winter identifica cuatro características principales de la teoría ortodoxa de la empresa, en cuya crítica se construye una parte significativa del programa de la TEE: $a$ ) una empresa es representada por una función de producción específica; $b$ ) las empresas son unidades racionales que maximizan el beneficio; c) operan en mercados de bienes homogéneos; y $d$ ) los arreglos contractuales internos son infalibles y no tienen costo alguno (Winter, 1996: 249-250).

En cuanto a la primera característica, la teoría neoclásica supone que todas las posibilidades técnicas eficientes de producción a un mismo nivel de output se encuentran

5 "Es posible definir la teoría neoclásica como una propuesta que tiene los siguientes atributos: 1) la suposición de agentes racionales con un comportamiento maximizador, con funciones de preferencias dadas y estables; 2) un punto de atención hacia estados de equilibrio alcanzados, $\mathrm{o}$ movimientos hacia estados de equilibrio; 3) la ausencia de problemas crónicos de información (hay, a lo sumo, un punto de atención en el riesgo probabilístico: excluyendo la ignorancia severa, la incertidumbre radical, o las percepciones divergentes de una determinada realidad). Notablemente estos tres atributos están interconectados.” Hodgson, 2007: 207. 
explícitamente reveladas en curvas de producción bien definidas, llamadas isocuantas (funciones continuas, cóncavas al origen), que señalan distintas combinaciones óptimas de recursos. En la medida que se alejan del origen, simbolizan mayores niveles de producción. El espacio geométrico donde están representados todos los niveles de producción se denomina función de producción, y muestra en qué medida aumenta el output ante incrementos infinitesimales de los factores productivos. Bajo estas condiciones de certeza técnica absoluta, el único objetivo racional que persigue la empresa es obtener el máximo beneficio.

Al respecto, la TEE propone que debido a la racionalidad limitada que caracteriza a las empresas, sólo pueden conocer una parte de la isocuanta, que dominan gracias a la práctica cotidiana; es decir, sólo reconoce algunas de las posibilidades técnicas en las cuales logra especializarse y adquirir capacidades distintivas. En cuanto la empresa aumenta su producción, pequeños tramos de otras isocuantas son revelados; de esta forma la unión de los distintos puntos eficientes de producción simboliza una senda de expansión tecnológica caracterizada por un comportamiento errático aunque ascendente.

En la TEE, esta senda de expansión expresa que el cambio técnico en una empresa individual ocurre de manera progresiva al explorar y descubrir los elementos útiles cercanos a su entorno. La empresa realiza una búsqueda local; en el sentido de que una distribución de probabilidad hipotética que mide la aparición de nuevas combinaciones se concentra primordialmente en técnicas cercanas a las que son conocidas. En suma, la empresa no puede representarse por una función de producción bien definida ya que no se conocen todas las posibles combinaciones técnicas de producción, sólo existe un conjunto de técnicas específicas que la empresa domina, en las cuales basa sus capacidades, y hay otras en un radio adyacente muy cercano a lo que ésta "sabe hacer", las cuales pueden ser adquiridas mediante exploración.

En lo que concierne al segundo inciso, la teoría neoclásica propone que la empresa funciona como un organismo homogéneo con racionalidad plena capaz de realizar una combinación eficiente de los factores de producción (trabajo y capital). Esto le permite establecer como única regla de decisión la captación del mayor beneficio posible o maximización de la ganancia (Nelson y Winter, 1974: 887; 1982: 53). La TEE establece que aunque la obtención de beneficio es muy importante para la empresa, la búsqueda de ganancia es sólo uno de sus diversos objetivos explícitamente reconocidos. De manera opuesta a la premisa de maximización de la ganancia, la TEE propone reglas de decisión o heurísticas particulares que conducen a las empresas a desarrollar actividades de "búsqueda de ganancia" o "esfuerzos motivados por la ganancia". Estas reglas son ejecutadas por agentes empresariales que no tienen certeza plena de todas las 
posibilidades a las que se enfrentan y que poseen capacidades cognitivas y de cálculo limitadas. En otras palabras, la maximización plena del beneficio es un supuesto irreal en un entorno caracterizado por la incertidumbre, donde la empresa debe poseer cierta capacidad de maniobra que le permita tomar decisiones ex post y resolver problemas de adaptación, derivados de cambios imprevisibles en su entorno.

La tercera característica de la teoría de la empresa neoclásica referida por Winter es suponer mercados de bienes homogéneos. Estos mercados se encuentran constituidos tanto por insumos productivos como por bienes de consumo. Presuponen preferencias transitivas que pueden ser expresadas en funciones continuas y bien definidas, por lo que no sólo los factores productivos sino también los bienes de consumo son susceptibles de divisiones infinitesimales. Los mercados se "vacían" a través de ajustes sistemáticos en los precios que ocurren de manera inmediata, en los que el factor temporal no juega ningún papel considerable ${ }^{6}$. El equilibro de mercado es la única solución posible a los movimientos de ajuste de las variables, y éste se alcanza cualesquiera que sean las condiciones iniciales de dotación de los recursos productivos.

Bajo las condiciones expuestas, el progreso técnico (y la ganancia extraordinaria que implica) es considerado una eventualidad que tiende a desaparecer en cuanto se produce cierta sucesión de ajustes de mercado. Al respecto, la TEE propone que la característica más importante del cambio técnico es su permanencia como condicionante del desempeño empresarial, al modificar no sólo la cantidad de bienes producidos sino, ante todo, la calidad de los mismos. Cuando esto ocurre, las empresas son capaces de modificar los precios relativos del mercado: de actores pasivos se convierten en motores del cambio económico, y también modifican los gustos y preferencias de los consumidores, así como las condiciones bajo las cuales se desenvuelve la producción. En este contexto, el equilibrio estático no tiene ningún sustento epistemológico más allá de un punto de partida analítico-hipotético.

Finalmente, la pertinencia del cuarto inciso radica en que introduce el aspecto organizacional ${ }^{7}$ en la discusión empresarial. Como señalan Nelson y Winter (1982:

6 El método expositivo de la teoría neoclásica es el de la estática comparativa, y opera mediante el cotejo de dos o más "momentos" que muestran el "movimiento" de las variables precio y cantidad ante ajustes en el mercado. Este método se opone radicalmente al de la TEE, cuyo objetivo es explicar los fenómenos sociales dentro de un continuum de cambios dinámicos en el tiempo.

7 Algunas interpretaciones de la función neoclásica de producción consideran que, además de los factores productivos K y L, existe un "factor organizacional", el cual representa los insumos empresariales y organizativos de la empresa. En una función Cobb-Douglas con la siguiente forma: $\mathrm{X}=b_{0} L^{b 1} K^{b 2}$, la variable $b_{0}$ representa un "parámetro de la organización técnica", e implica claramente que aun si dos empresas poseen las mismas cantidades de factores 
54-57) y Winter (1996: 252), el sustento inherente de la maximización racional del beneficio es el supuesto de contratos internos perfectos e infalibles, que presupone la unanimidad de intereses entre los accionistas de la empresa, y la ausencia de cualquier tipo de conflicto. Cuando se analiza el funcionamiento interno de la empresa desde la perspectiva de la teoría neoclásica "se supone que se alcanza fácilmente, mediante el intercambio voluntario, una relación plenamente cooperativa entre los diversos intereses económicos organizados en la empresa, y se supone que esto se logra en forma rutinaria aunque implícita" (Winter, 1996: 252). Las complejidades teóricas derivadas del conflicto y de la cooperación se encuentran completamente ausentes en el análisis neoclásico.

Del análisis de los puntos señalados por Winter (1996), puede inferirse que en la teoría económica ortodoxa la empresa transita por un paisaje libre de cualquier tipo de fricción derivada tanto de adecuaciones tecnológicas (aprendizaje, propiedad intelectual, procesos de innovación, etcétera), como de problemas organizacionales (incentivos, desigualdad en el procesamiento de información, etc.). De ocurrir cualquier tipo de fricción interna puede preverse que se degrade la viabilidad de una rentabilidad máxima y resulte inviable cualquier punto de equilibrio.

En suma, uno de los grandes méritos de la TEE consiste en despejar el camino y establecer los pilares de una teoría económica de la empresa alejada de los principios tradicionales de maximización de la ganancia y de equilibrio interno. La TEE ha demostrado que el análisis de los procesos relacionados con estrategias basadas en conocimiento imperfecto y la estructura organizacional no se encuentra presente en la discusión neoclásica de la empresa. En respuesta, la TEE intenta establecer un concepto de empresa desde una perspectiva dinámica, que le permita considerar cambios adaptativos internos que respondan a las variaciones del entorno de selección, así como transitar por un sendero de expansión técnica y organizacional, derivado del aprendizaje adquirido en la praxis cotidiana. A continuación, se analizará hasta qué punto la TEE ha avanzado en la comprensión de los procesos empresariales internos.

productivos, distribuidos de la misma manera y con los mismos rendimientos a escala, la forma particular en que una empresa es capaz de organizar la producción podría modificar la cantidad producida (mas no la calidad). Ésta es una interpretación menos rígida del funcionamiento de la empresa en la teoría neoclásica, e incluso podría considerarse como un reconocimiento débil de heterogeneidad empresarial, empero, sus consecuencias teóricas no han sido suficientemente exploradas por la economía ortodoxa. Véase Koutsoyiannis, 2002. 


\section{La empresa como depósito de conocimiento}

La TEE reconoce que los sistemas agregados se encuentran conformados por una gama muy amplia de agentes empresariales, con características propias que los distinguen entre sí. La definición más general de empresa propuesta por la TEE se basa precisamente en lo que considera el elemento más importante de la diversidad empresarial: el conocimiento. Propone una definición amplia de empresa que la identifica como un depósito de conocimiento productivo útil en un determinado momento. Cada empresa se caracteriza por tener un "intervalo muy específico de conocimiento productivo (...) que a menudo involucra características idiosincráticas que la distinguen incluso de las empresas superficialmente similares en la misma línea (o líneas) de actividades" (Winter, 1996: 264). Lo que la empresa es capaz de producir en un intervalo de tiempo dado, no depende tanto de la disponibilidad de recursos productivos o de la forma óptima de combinarlos (del estado técnico, como supone la teoría neoclásica), sino del stock de conocimiento acumulado . $^{2}$

No obstante que los depositarios naturales del conocimiento son los agentes individuales, cuyos órganos cerebrales son capaces de desarrollar las funciones de almacenamiento, procesamiento y transmisión del mismo, Nelson y Winter (1982) proponen un énfasis especial en la importancia del conocimiento organizacional como un atributo de la "empresa como un todo", el cual no se constituye como una suma o agregación simple del conocimiento de los agentes individuales, sino que representa un conocimiento distinto e identificable de manera independiente. Debido a que un solo agente es incapaz de conocer todas las tareas que se realizan al interior de una empresa y la forma en que cada una de ellas se relaciona con el todo (aun aquellos que se desempeñan en labores ingenieriles o gerenciales), la organización como un ente unificado "sabe" más que cualquier agente individual. Eso significa que cualquier agente es reemplazable y puede ser separado de la empresa sin daños relevantes que impidan su funcionamiento (Nelson y Winter, 1982: 59-65).

Parte de lo que la empresa "sabe", es decir, del conocimiento que posee, incluye la habilidad necesaria de coordinar todas las unidades de tarea y departamentos internos. El conocimiento organizacional actúa a través de toda la estructura empresarial,

8 Una de las referencias obligadas que fundamenta la idea de empresa basada en el conocimiento, es el artículo clásico de Arrow (1962), donde propone un marco conceptual que considera los cambios en la cantidad de conocimiento usado en la producción como una variable endógena al modelo de crecimiento de la empresa, rompiendo hasta cierto punto con la rigidez de la teoría neoclásica. 
distribuyendo adecuadamente distintos tipos de habilidades de acuerdo con su funcionalidad particular y espacial. La TEE supone un tránsito fluido de información entre todas las acciones necesarias para completar el proceso productivo. Las habilidades de coordinación de la empresa en su conjunto y el conocimiento específico de cada unidad de tarea no pueden ser enteramente codificados en manuales operacionales, por lo que las principales partes de dicho conocimiento resultan intraducibles en otros contextos, incluso entre distintas unidades pertenecientes a la misma empresa.

El conocimiento así descrito se convierte en un límite del crecimiento de la empresa y un factor que puede modificar su forma jerárquica, debido a que la factibilidad de realizar algún proceso productivo en un momento dado depende, en última instancia, del conocimiento que ésta posea. El conocimiento organizacional adquiere una constitución moldeable, que evoluciona a través de senderos específicos de interacción. Nelson y Winter establecen que el conocimiento adquirido por una organización puede ser modificado de manera deliberada por medio de esfuerzos conscientes dirigidos a alcanzar algún fin particular, o mediante acciones no deliberativas resultado de la acción automática de los agentes. El conocimiento empresarial puede incrementarse también cuando los agentes internos "aprenden haciendo" (learning by doing) formas más eficientes de realizar sus tareas cotidianas. De la misma forma, es posible perder conocimiento cuando los agentes olvidan los detalles de las tareas que habían desempeñado habitualmente. Todos estos procesos de adquisición y modificación de conocimiento ${ }^{9}$ pueden resultar o muy costosos o relativamente fáciles de llevar a cabo según sea el caso (1982: 64); sin embargo, para la TEE tienen una característica común: son resultado primordial de una actividad automática y no deliberativa realizada por los agentes involucrados.

En suma, en la TEE el conocimiento es la característica primordial que define a la empresa y le da identidad propia dentro de la microdiversidad que conforma los sistemas económicos agregados. La empresa individual es un ente capaz de desarrollar procesos

9 Al respecto, Cohen y Levinthal (1989) proponen que además del conocimiento automático por medio del cual la empresa se vuelve más eficiente en lo que sabe hacer, definido por el concepto de learning by doing, existe otra forma en la que puede adquirir nuevos conocimientos, que se basa en el desarrollo de capacidades de absorción. La empresa no sólo invierte recursos en acrecentar o modificar el conocimiento que posee, también lo hace para tratar de apropiarse del conocimiento generado tanto por otras empresas como por instituciones públicas de investigación y desarrollo. Incluso, los autores afirman que la asimilación de oportunidades tecnológicas depende, en gran medida, de la asimilación del conocimiento externo. La empresa, por tanto, no sólo desarrolla capacidades para explotar el conocimiento que posee sino para explorar el ambiente y captar nuevas posibilidades. 
propios de aprendizaje y de retener el conocimiento que adquiere para mejorar el desempeño de sus actividades. Pero ¿cómo se genera nuevo conocimiento? La mayor parte del conocimiento se logra por el simple hecho de hacer lo que ya se conoce; y cuando surge algún acontecimiento que requiere nuevas prácticas, o si se quiere explorar nuevas combinaciones, la búsqueda de posibilidades se limita a un área local o adyacente a lo que ya se conoce. El conocimiento así adquirido se concretiza en ciertas capacidades que le confieren elementos distintivos de desempeño y ventajas competitivas sobre las demás empresas. En consecuencia, la empresa como unidad de análisis y no los agentes individuales que la integran, es la entidad que "sabe" cómo hacer las cosas ${ }^{10}$. La elección automática entre distintas opciones y la ejecución de nuevas tareas constituye una parte esencial de las capacidades de la empresa como un agente propio.

Este planteamiento es muy controversial y al parecer insuficiente para comprender la actividad de la empresa en un entorno competitivo. Basar la conducta de los agentes empresariales únicamente en un comportamiento automático, los despoja de su principal característica cognitiva: la posibilidad de generar soluciones distintas a problemas ya existentes, y soluciones nuevas para problemas inesperados (Mantzavinos, 2001). Como apunta Vromen (2006), el aspecto cognitivo empresarial se conforma más bien por un contexto de coexistencia permanente entre las acciones derivadas de la elección consciente, y de las que son inconscientes o rutinarias; en algunas ocasiones incluso las actividades automáticas se encuentran precedidas de una elección consciente. Más aún, como plantea Antonelli (2008), la generación de innovaciones en la empresa tiene que ver con respuestas creativas (deliberativas) que los agentes asumen ante cambios en su entorno, más que con un aprendizaje pasivo y rutinario derivado de las rutinas organizacionales. La concepción evolutiva del conocimiento empresarial resulta inadecuada para comprender el accionar dinámico de las empresas en el mercado.

\section{La empresa como unidad de análisis}

Es pertinente exponer con más detalle la idea de que la empresa funciona como un organismo unificado en la toma de decisiones y en sus procesos internos. Al respecto, a pesar de sus marcadas diferencias, la TEE mantiene coincidencias metodológicas importantes con la teoría neoclásica ortodoxa; una de ellas parece ser precisamente el "buscar capturar con unos cuantos supuestos simples las fuerzas motivacionales

10 Winter establece que "son las empresas, no las personas que trabajan para las empresas, quienes saben cómo fabricar gasolina, automóviles y computadoras" (1996: 258). Resulta poco creíble que un sistema social pueda "saber" algo, en cuanto la atribución del saber corresponde a los individuos. En todo caso, lo que puede ocurrir es que un sistema social cultive, potencie y aproveche los saberes de los agentes individuales. 
operacionalmente más consistentes y poderosas que tienden a modificar el comportamiento de la empresa como un todo" (Nelson y Winter, 1982: 58).

Como se ha visto, una de las razones más poderosas que comprueba la insuficiencia analítica de la teoría neoclásica es precisamente su caracterización de la empresa como un agente unificado sin conflictos internos. No obstante, habiendo revelado el problema, la TEE busca retomar dicha simplificación al pretender definir a la empresa sólo a partir de sus características colectivas, omitiendo cualquier fricción interna. Incluso la descripción que hace Winter al referirse a la manera en que la teoría neoclásica concibe los procesos internos de la empresa, pudiera muy bien coincidir con el punto de vista de la TEE: "Allí se supone que se alcanza fácilmente, mediante el intercambio voluntario, una relación plenamente cooperativa entre los diversos intereses económicos organizados en la empresa, y se supone que esto se logra de forma rutinaria aunque implícita" (Winter, 1996: 252). Más adelante se verá que esta definición concuerda literalmente con el concepto de rutina organizacional que propone la TEE.

Dentro del contexto de la empresa como unidad, y llevando aún más lejos la metáfora de que "sabe" lo que debe hacer, la TEE compara la conducta de la empresa con la de los individuos al afirmar que "el comportamiento de una organización es, en sentido limitado pero importante, reducible al comportamiento de los individuos que son miembros de esa organización"11 (Nelson y Winter, 1982: 72). Una de las características más importantes que se le confiere al agente individual en función de la analogía propuesta, es poseer habilidades específicas para desarrollar sus actividades. La habilidad individual se define como "una capacidad que se relaciona con una secuencia lisa de comportamientos coordinados los cuales son ordinariamente efectivos en el logro de sus objetivos, dado el contexto en el que normalmente ocurren" (ibid., 73). Dichas habilidades se encuentran conformadas por dos tipos básicos de conoci-

11 En éste como en otros pasajes, la TEE recurre a la analogía para no explicar la ontología de sus conceptos. Aun cuando toda la discusión se concentre en el fundamento epistemológico de la empresa como agente dentro de sistemas agregados, se introduce la analogía entre el sistema y el agente individual para explicar el pilar más importante de su propuesta teórica: la rutina organizacional. Nada se dice de cuál es el "sentido limitado" de esta comparación, peor aún, no se diferencian epistemológicamente los distintos niveles de emergencia entre los individuos y la empresa, por lo que resulta confuso definir las propiedades de la empresa en función de las características de los individuos. Por recurrir a otra analogía, esto equivaldría a definir al ser humano como un sistema formado por órganos vitales específicos y, paralelamente, definirlo también sólo por su capacidad de pensar, reduciendo su plano ontológico a una sola de las funciones de uno solo de sus órganos. 
miento: una parte explicita que puede ser transmitida a los demás agentes sin pérdida de información; y una parte tácita que no es codificable, muy difícil de transmitir.

$\mathrm{Al}$ afirmar que "la empresa sabe lo que tiene que hacer", es claro que la propuesta de la TEE apunta primordialmente hacia una especie de conocimiento organizacional tácito, que no es codificable y que no radica en ningún lugar físico concreto. De lo contrario, afirmar que la empresa como unidad posee algún tipo de conocimiento producto del racionamiento metódico y deliberado, que anticipe en alguna medida las futuras consecuencias de sus acciones, implicaría atribuirle propiedades características de los seres vivos, lo cual exigiría especifcar de la ubicación de ese conocimiento y de cuáles son los "órganos empresariales" capaces de procesarlo ${ }^{12}$. Para evitar estas complicaciones, la empresa se define como un agente autómata, capaz de realizar todas las actividades productivas necesarias y coordinarlas de la mejor manera posible sin que nadie, a ciencia cierta, esté al tanto de cómo lo logra ${ }^{13}$. La organización trasciende al conocimiento individual o a cualquier tipo de artefacto físico capaz de almacenarlo y procesarlo ${ }^{14}$.

12 Al respecto argumenta Vromen: “¿Pero una organización, concebida como una especie de entidad sui géneris con comportamiento propio, puede en realidad desarrollar una deliberación consciente? Al parecer, las organizaciones como un todo no pueden hacerlo. Parece ser que la elección deliberada no es un atributo de las organizaciones como un todo. Las organizaciones no son agentes como los individuos, los cuales tienen la opción de desarrollar una elección consciente y deliberada o seguir una regla de manera inconsciente (o subconsciente) y automática" (2006: 6).

13 Dosi, Faillo y Marengo, al especificar el concepto de rutina establecen que "éste no implica ninguna presunta consideración de algún propósito evidente: una de las cosas más interesantes acerca de las rutinas es realmente que ellas se encuentran a menudo en contextos donde nadie puede explicar lo que son, excepto en términos vagos como 'la manera en que las cosas son hechas por aquí' (2003: 6).

14 Conviene situar correctamente la importancia crucial que tiene el conocimiento colectivo en los procesos empresariales. De acuerdo con Vromen (2006), sería un error intentar reducir el conocimiento sólo a lo que ocurre dentro de la cabeza de los individuos: los aspectos sociales y culturales tienen una importancia decisiva en el proceso cognitivo. El problema es que la TEE sólo reconoce un tipo de conocimiento social tácito, pero ¿la empresa sólo posee conocimiento tácito?, es claro que no, existe también conocimiento procedimental muy detallado en las labores productivas complejas. Vromen (2006) otorga gran importancia a los artefactos que se utilizan durante el proceso productivo al ser una actividad grupal, como extensiones compartidas del conocimiento individual (un lugar físico de almacenamiento son las computadoras, por ejemplo). La TEE no niega la importancia de los artefactos, pero sí demerita su utilidad. 


\section{Las rutinas organizacionales, fundamento epistemológico de la empresa en la TEE}

Concretamente, la metáfora de que la empresa "sabe" cómo hacer las cosas, conduce a la formulación del concepto de rutina organizacional, indispensable para explicar su accionar interno ${ }^{15}$. Becker (2004: 643), por ejemplo, establece que una de las aportaciones más importantes de la TEE es haber reintroducido en el debate económico la importancia de las rutinas organizacionales como una forma de explicar el funcionamiento de la empresa y de la economía en su conjunto, a pesar de las ambigüedades e inconsistencias del concepto ${ }^{16}$. Incluso Becker et al. (2005: 775) sostienen que las rutinas son el componente fundamental del comportamiento organizacional y depositarias de las capacidades organizacionales, por lo que el análisis de los cambios empresariales generados por variaciones internas en los elementos constitutivos se reduce a cambios en las rutinas organizacionales. Puede afirmarse que la TEE de la empresa descansa esencialmente en la idea de que las rutinas organizacionales son los "depósitos" y generadores de una gran parte del conocimiento útil en el proceso productivo. En suma, las rutinas organizacionales representan la "memoria organizacional" de la empresa (Nelson y Winter, 1982: 99).

El concepto de rutina se construye a partir de la analogía descrita arriba entre habilidades individuales y "habilidades organizacionales". Dicha analogía se fundamenta en algunas características compartidas entre las actividades de la empresa y las de los individuos: 1) las rutinas, al igual que las habilidades, son programáticas en el sentido de que involucran una secuencia de pasos que se desenvuelven invariablemente uno detrás de otro; la ejecución de cada uno induce de manera involuntaria la realización del siguiente, y así hasta terminar por completo la tarea; 2) debido a que el conocimiento esencial en el que se basan las labores empresariales es tácito, tanto las habilidades individuales como las rutinas organizacionales se activan a partir de acciones no deliberativas, lo cual se expresa en el hecho de que al efectuar una tarea,

15 "Y esto, desde luego, es el corazón de nuestra propuesta teórica: el comportamiento de las empresas puede ser explicado por las rutinas que ellas emplean. El conocimiento de las rutinas es el corazón en la comprensión de su comportamiento. Modelar la empresa significa modelar sus rutinas y la manera en la que cambian a través del tiempo." (Nelson y Winter, 1982: 128.)

16 Vromen (2006) plantea que dicha ambigüedad se deriva fundamentalmente del hecho notable de que la definición del concepto abarca cada vez más propiedades y funciones, a tal grado de cubrir bajo su enunciación prácticamente cualquier actividad que ocurra al interior de la empresa. El uso indiscriminado del término que tiende a explicar prácticamente todo, convierte a la rutina en un concepto sin contornos claros, y epistemológicamente no-autocontenido. 
los detalles de la misma quedan fuera de las consideraciones cognitivas de quien la realiza, ya sea un agente individual o una empresa; y 3) las acciones son efectuadas de manera automática y sin conciencia plena de lo que se está haciendo; es decir, aun cuando la acción implique cierta elección, las habilidades individuales tanto como las rutinas empresariales "dirigen" las decisiones de manera automática, sin necesidad de recurrir a ningún tipo de deliberación consciente (Nelson y Winter, 1982: 73).

Desde la perspectiva de la TEE, todo lo que se requiere para que las rutinas organizacionales dominen los procesos internos en una empresa e induzcan una cooperación armónica, es que los agentes que la constituyen sigan "conociendo sus trabajos" (Nelson y Winter, 1982: 100), que continúen haciendo lo que saben hacer. Sin embargo, puesto que en opinión de la TEE, tanto los agentes individuales como las organizaciones se caracterizan por tener racionalidad limitada, permanece latente en esta propuesta una mínima exigencia de coordinación entre las tareas de distinta naturaleza que realiza cada individuo dentro de la organización. ¿Cómo se resuelve el problema de coordinación bajo el dominio de las rutinas al interior de la empresa?:

Un flujo de mensajes llega a la organización procedente del ambiente (...). Los miembros de la organización reciben esos mensajes y los interpretan recurriendo al desempeño de su repertorio de rutinas. Incluye algunos que pueden ser interpretados como directamente productivos (...) y otros de naturaleza administrativa o de procesamiento de la información. (...) En cualquiera de los dos casos, tanto como una consecuencia incidental de otro tipo de acciones o como actos deliberados de comunicación, la ejecución de las rutinas por cada miembro de la organización genera una corriente de mensajes hacia los otros. Estos mensajes son interpretados gracias a ciertas acciones que realizan sus destinatarios, quienes generarán otras acciones, mensajes, interpretaciones, etc. (...) Existe, por tanto, en la operación de las rutinas, una corriente circular interna de información en equilibrio al interior de la organización (...) (Nelson y Winter, 1982: 103).

Por lo tanto, los agentes individuales, al tiempo que realizan sus tareas cotidianas (o por el hecho de realizarlas), son capaces de percibir e interpretar correctamente un conjunto de mensajes que provienen tanto de otros individuos como del ambiente que les rodea. La información captada sensorialmente se combina con el conocimiento previo que poseen, parte del cual (el más importante) se encuentra contenido en las rutinas personales que han logrado formar. Existe un flujo continuo de información al interior de la empresa que se nutre constantemente con las señales del medio ambiente y con los mensajes que generan los propios agentes; este flujo continuo es de fácil acceso para todos los individuos, y cada miembro recoge la información necesaria 
que le permite desempeñar sus labores cotidianas. De esta forma, cada agente es capaz de armonizar su propio proceso rutinizado de actividad con el de la empresa, contribuyendo a solucionar el problema de coordinación con el simple hecho de "hacer lo que sabe".

Es claro que si se desea un funcionamiento adecuado de la empresa, el proceso descrito ha de fluir sin contratiempos, por lo que todos los agentes deben interpretar correctamente la información que perciben del exterior, o lo que es lo mismo, deben poseer capacidades muy cercanas a un estándar de homogeneidad cognitiva. Se propone que dicho principio de homogeneidad cognitiva existe en la TEE, no porque se pretenda afirmar que en ella no se encuentra un reconocimiento de la heterogeneidad cognitiva de los agentes que intervienen en un contexto empresarial, sino porque, al parecer, esa heterogeneidad se desvanece paulatinamente gracias al funcionamiento de las rutinas organizacionales. En concordancia con la TEE puede afirmarse: $a$ ) dos agentes con distintas capacidades cognitivas y de procesamiento de la información interpretarán de la misma manera los mensajes que lleguen a un mismo puesto de trabajo, ocupado en distintos periodos por cada uno de ellos; y $b$ ) dos agentes que realicen una misma labor, en un mismo puesto de trabajo y durante un mismo periodo interpretarán exactamente de la misma forma los mensajes que llegan del medio ambiente. En otras palabras, se presume un proceso de trabajo perfectamente estandarizado, en el que los agentes se desempeñen como estructuras funcionales del proceso productivo, reemplazables en cualquier momento.

¿Cómo se logra la homogeneidad cognitiva ex post? Nuevamente: “...lo que es central para la coordinación es que los miembros individuales, conociendo su trabajo, interpretan y responden correctamente los mensajes que reciben" (Nelson y Winter, 1982: 104). Al parecer, otra de las virtudes que poseen las rutinas organizacionales es proporcionar una interpretación funcionalmente mecánica de la realidad en la que se desenvuelve el proceso productivo, en el sentido de que la "elección" de la interpretación correcta de los mensajes exteriores por parte del agente se basa completamente en el "repertorio" previo de rutinas personales que éste posee; es decir, es una elección automática que no necesita ningún tipo de reflexión consciente.

¿Es sostenible esta interpretación basada en la coordinación armónica y automática al interior de la empresa y cierta propensión hacia la homogeneidad cognitiva? La evidencia empírica basada en la teoría del aprendizaje parece contradecir la propuesta evolutiva. Un ejemplo de ello es el modelo que presenta Kitcher (2001: 94-97) respecto del proceso cognitivo individual. Cuando un estímulo impacta en la conciencia inmediata del sujeto a través de la percepción, la memoria de trabajo realiza 
varias "búsquedas" internas en distintos "compartimentos" cerebrales. No obstante que todos los agentes dentro de un contexto empresarial específico se encontraran sometidos a los mismos estímulos del ambiente durante toda su vida y compartieran las mismas metas de largo plazo, la representación particular de la realidad que cada uno de ellos construiría en sus "memorias" declarativa y procedimental sería distinta, ya que cada uno interpretaría y almacenaría de forma diferente las proposiciones que derivan de los estímulos percibidos. No existe ninguna evidencia concreta de que las "búsquedas" que los individuos realizan en sus distintos "compartimientos" cerebrales, cuando éstos tienen que resolver algún problema específico, recuperen exactamente las mismas proposiciones inferenciales con base en las cuales establecerán su práctica cotidiana.

La interpretación que ofrece la TEE acerca de que los mensajes que fluyen a través de las rutinas organizacionales son entendidos correctamente por los agentes individuales, simplemente por el hecho de saber hacer lo que hacen, es insostenible en el marco analítico anterior. La TEE no explica de qué manera las rutinas organizacionales contribuyen a que todos los individuos desarrollen capacidades certeras de búsqueda interna y de recuperación de los elementos precisos que les permitan interpretar correctamente la información, o si son capaces de interrumpir dicho proceso deliberadamente y de alguna forma operar sin él; más aún, no explica de qué manera concreta es posible evitar errores de interpretación e interferencias en la transmisión de los mensajes.

El proceso de aprendizaje individual es incompatible con la operación de rutinas organizacionales tal y como las concibe la TEE. La metáfora de un agente que realiza su praxis cotidiana de modo automático y no deliberativo no coincide con la realidad. Al parecer, en su intento de crítica a la teoría neoclásica, la TEE evolutiva se ha concentrado en el otro extremo de la racionalidad plena, al proponer un agente totalmente pasivo. Sin embargo, es pertinente reconocer que el comportamiento basado en rutinas puede representar una parte (mayor o menor según ciertas características) de la práctica de los agentes empresariales; lo que se pone en duda es la posibilidad de que todo tipo de decisión y comportamiento dentro de la empresa se explique completamente por la interacción de actividades no deliberativas e inconscientes.

\section{Solución de conflictos internos}

A pesar de que la TEE afirma que su intención se encuentra lejos de ignorar la existencia de posibles elementos divergentes entre los intereses de los miembros de la empresa, lo cierto es que las consideraciones previas se encuentran muy cercanas a una presunción de intereses convergentes. Aun si existieran conflictos internos oca- 
sionados por algún tipo de disparidad de intereses, las rutinas organizacionales por sí mismas parecen capaces de absorberlos y enmendarlos sin ningún contratiempo para la funcionalidad de la empresa. Por lo tanto, en la medida en que el conflicto no representa ninguna complicación especial, la TEE acepta que aunque los agentes interpreten correctamente los mensajes que reciben de su entorno, ello no implica que "hagan lo que ellos han dicho que harían", es decir, existe un riesgo de que los miembros "hagan lo que saben hacer" recurriendo a distintos niveles de oportunismo.

Incluso si un agente llegara a violar los estándares de comportamiento establecidos por las empresas, no necesariamente se alterarían las expectativas generales que se tienen sobre la operación de toda la planta en su conjunto. No significa tampoco que haya algún tipo de "consecuencias para la producción que sean inconsistentes con los resultados estadísticamente estables y se encuentren dentro del rango esperado" (Nelson y Winter, 1982: 108). La TEE se encuentra permeada de un fundamento epistemológico colectivista, al reducir todos los movimientos de los agentes individuales a una sencilla evaluación estadística de rangos probabilísticos, donde todas las influencias individuales que pudieran ejecutarse sobre el sistema en su conjunto se anulan mutuamente.

Otra forma de evitar conflictos potenciales entre los agentes, derivados de su casi inexistente disparidad de intereses, es mediante la formalización de las reglas que existen previamente de manera implícita en las rutinas, las cuales se transforman en normas de conducta ejecutables por una autoridad, mediante un código de sanciones que imponen estímulos negativos a su conducta oportunista. Los agentes que operan como elementos de control y sanción (managers) también realizan sus actividades con base en rutinas específicas. Los mecanismos formales e informales de control que posibilitan el cumplimiento de las normas, si bien son importantes para asegurar en algún sentido el funcionamiento de la empresa, tienen una influencia muy limitada comparados con la operación corriente de las rutinas. El proceso productivo como un todo orgánico y armónico basa una mínima parte de su funcionalidad en los problemas de agencia. En realidad estos no contribuyen en la solución de ningún problema sustancial que no haya sido resuelto antes por las rutinas organizacionales.

La solución de los problemas derivados del surgimiento de alguna actitud oportunista o de cualquier conflicto de intereses que tendiera a poner en riesgo la operatividad de la empresa, se encuentra presente en las rutinas organizacionales. En especial en su atributo de operar armónicamente todo el proceso productivo y, al hacerlo, establecer una tregua organizacional entre los agentes individuales involucrados. En el proceso productivo que opera con base en rutinas, los conflictos encuentran senderos predecibles de ocurrencia, que no afectan el funcionamiento esperado de la organización. Como afirman Nelson y Winter: 
En suma, la operación con base en rutinas implica una tregua completa entre los conflictos intraorganizacionales. Existe una tregua entre el supervisor y los supervisados en todo nivel de la jerarquía organizacional: se realiza la cantidad usual de trabajo, las reprimendas y los cumplidos ocurren con la usual frecuencia, y no se presenta ninguna exigencia para modificar de modo importante los términos de la relación. Hay una tregua similar entre los ejecutivos de alto nivel en la lucha por obtener promociones, poder y beneficios extra (1982: 110).

Resulta claro que la idea de rutinas organizacionales como tregua entre los distintos intereses individuales en la empresa puede interpretarse también como una propuesta de forma de gobierno basada en la confianza mutua y en la operatividad armónica de todos los procesos internos.

\section{Influencia de la incertidumbre en las rutinas organizacionales}

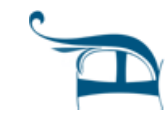

Queda aún pendiente la explicación de cómo la empresa puede enfrentar con éxito la incertidumbre que prevalece en los cambios externos. La TEE acepta que el ambiente en el cual se reproduce un sistema es cambiante y en algún sentido impredecible, por lo que es necesario establecer la manera concreta en que la empresa interpreta y se adapta a esos cambios. Dicha teoría considera que la empresa es un sistema abierto, que puede ser perturbado por las variaciones que ocurren en su entorno. Por tal motivo, aun cuando las rutinas organizacionales aseguren un funcionamiento armónico al interior de la empresa, éstas no pueden ser estáticas ni asegurar una funcionalidad perpetua. Es factible entonces introducir un parámetro de discontinuidad en el desempeño organizacional resultado de los cambios externos. Ejemplo de un posible cambio inducido por el ambiente en el contexto organizacional es el que se produce por la modificación en las condiciones de precio o calidad de los inputs de mercado, situación que obliga a la empresa a modificar sus patrones internos de producción. Otro ejemplo es el despido de personal que posee un conocimiento altamente contextualizado, adaptado a las rutinas vigentes y sin el cual la empresa podría enfrentar problemas de coordinación. Estos y otros posibles problemas pueden ser abordados y neutralizados por elementos de control interno (managers) que tiendan a "conducir" las rutinas hacia caminos menos adversos.

Los elementos de control pueden solucionar mediante adecuaciones internas los problemas de adaptación que surjan en el contexto organizacional, "el tiempo y los cambios en el ambiente sacuden a la organización con potenciales mutaciones, en contra de las cuales luchan sus sistemas de control" (Nelson y Winter, 1982: 117). Sólo aquellos acontecimientos que logren traspasar las barreras internas de control 
impuestas por la empresa, pueden generar cambios que modifiquen incrementalmente el curso de las rutinas vigentes. Existen rutinas para cambiar las rutinas.

La TEE oscila entre el establecimiento de un escenario organizacional en el que las rutinas como conocimiento no deliberativo representan la garantía más eficaz del transcurso armónico de todas las tareas al interior de la empresa, y otro punto donde es necesaria la incorporación de "elementos de control" que, de ser necesario, conduzcan correctamente la secuencia lisa por la que transcurren las rutinas. Se sugiere que todo aquello que escape del control automático de las rutinas será sometido a un control basado en el conocimiento del mánager. Esta concesión a las teorías del strategic management supone, como afirman Levinthal y March (1993), un agente organizacional específico con objetivos bien definidos y una capacidad inmejorable de procesar la información que obtiene del medio ambiente. Además, dicho agente sería capaz de condicionar las acciones de todos los demás agentes y controlar su ejecución. Levinthal y March (1993) afirman que conceder ante la idea del strategic management implica volver a una concepción de racionalidad que ahora recae en un solo agente, el cual posee grandes capacidades cognitivas y preferencias estables.

Es evidente que la economía evolutiva no encuentra una respuesta clara al interrogante central de cómo se gestan cambios importantes en el seno de un proceso productivo dominado por rutinas. La idea de rutinas que modifican rutinas no encuentra ningún asidero epistemológico o empírico. Al parecer, los procesos de cambio discontinuo por los que atraviesan las empresas, sobre todo en contextos de reestructuración productiva general, no pueden ser explicados por esta idea. El concepto de rutina implica un desequilibrio entre las actividades de explotación y exploración en el sentido de March (1991), donde la empresa se dedica a explotar sus capacidades adquiridas, descuidando las actividades de monitoreo del mercado, lo que le resta posibilidades de éxito futuro y de anticipación a las oportunidades futuras.

\section{Conclusión}

La teoría económica evolutiva significó un avance conceptual y explicativo en relación con la teoría de la empresa establecida por la economía neoclásica. El cambio sustancial en los modos de referencia se expresa en la necesidad de estudiar los procesos empresariales internos, concretamente los procesos cognitivos y organizacionales. Existen dos modalidades de progreso explicativo en la TEE en relación con la teoría ortodoxa: 1) la eliminación de esquemas previos incorrectos, concretamente del principio de racionalidad plena y sus consecuencias analíticas, que afecta todos los niveles de desempeño empresarial; 2) la incorporación de nuevos esquemas, principalmente la importancia del conocimiento productivo. 
A la maximización racional de las oportunidades técnicas de producción, la TEE opone una búsqueda local de soluciones, basada en las capacidades cognitivas que la empresa va forjando en la medida que realiza sus actividades cotidianas. La adquisición de capacidades cognitivas se apoya principalmente en la experiencia, en lo que la empresa ha aprendido a hacer. Estos procesos generalmente son irreversibles y acumulativos.

En cuanto la TEE ha identificado al conocimiento como factor primordial de la producción, plantea que la empresa como agente agregado "sabe" más que cualquier agente individual; es decir, el conocimiento empresarial presenta un marcado sesgo colectivo como característica principal. El conocimiento empresarial, definido como un atributo colectivo, es primordialmente tácito y no deliberativo. En estas dos ideas se concentra la dirección precisa que la TEE busca dar al aspecto cognitivo empresarial.

La revisión crítica de la teoría neoclásica realizada por la TEE se enfoca fundamentalmente en proponer que el conocimiento es la base del desempeño empresarial y la característica fundamental de su definición. La TEE define a la empresa de acuerdo con un solo tipo de conocimiento: el que se encuentra almacenado en las rutinas organizacionales.

La idea implícita en la propuesta evolutiva de la empresa es que las rutinas son, al mismo tiempo, elementos generadores de conocimiento y la principal forma de solución de problemas. En síntesis: la empresa es lo que sus rutinas son (Nelson y Winter, 1982). Pero ¿cuál es el contenido explicativo de este supuesto?, es decir, ¿cómo se definen las rutinas organizacionales? La única referencia que puede encontrarse en Nelson y Winter (1982), es que las rutinas son a la organización lo que las "habilidades" son al individuo. Esta formulación tiene poca consistencia lógica.

Si la empresa es lo que son sus rutinas, y a su vez, las rutinas son equiparables con las habilidades de los individuos, ¿cómo se definen las habilidades individuales? No se encuentra una definición consistente de las características de los agentes que conforman a la empresa, ya que no se considera necesaria una teoría del agente individual. Por lo tanto, la TEE intenta dar respuesta al cuestionamiento anterior, recurriendo a una explicación de cómo actúan o se manifiestan las habilidades individuales. Se plantea que son comportamientos programáticos, no deliberativos y automáticos. En ningún lugar se especifica lo que son en realidad las habilidades individuales, más allá de algunos comportamientos visibles que generan.

Afirmar que las habilidades son comportamientos con ciertas características, no refiere nada sobre las características de los agentes que constituyen la empresa. Tampoco se recurre a una definición de agente individual que sustente esta analogía, sólo se encuentra una vaga alusión al agente, de donde se infiere que éste $e s$ lo que son sus 
habilidades, y estas habilidades se caracterizan por ser programáticas, no deliberativas y automáticas. Al no sustentarse lógicamente la idea de que la empresa es lo que son sus rutinas, y su derivación referente a que las rutinas "son" lo que las habilidades para el individuo, es cuestionable que el concepto rutina represente un verdadero progreso conceptual o explicativo en la teoría de la empresa.

La "caja negra" de la organización interna queda parcialmente abierta al no postular ningún elemento significativo y consistente en cuanto a la constitución e interrelación de los elementos internos. La empresa como sistema encuentra una explicación holística en la TEE (véase Auyang, 1998), al construir referentes teóricos que atienden sólo la parte agregada del fenómeno y, a partir de ello, tratar de explicar algunas características vagas de los elementos que la constituyen.

En contraste, la apuesta fundamental de este trabajo radica en develar los fundamentos de una teoría de la empresa en dos niveles emergentes. Por un lado, el nivel de los microcomponentes, que comprende a los agentes individuales que conforman a la empresa, y las microestructuras de interrelación que desarrollan a partir de su praxis cotidiana; por el otro, el nivel de los componentes agregados, referente a los fenómenos colectivos que surgen a partir de las interacciones de los componentes.

Ninguno de los dos niveles es reductible al otro, por lo que cada uno presenta una independencia relativa y características propias. No obstante, la construcción de una teoría de la empresa que atienda su naturaleza ontológica debe concentrase no sólo en explicar las características de ambos niveles sino, sobre todo, en las particularidades derivadas de su interrelación.

A la fecha existen teorías parciales de la empresa; algunas intentan explicar únicamente los fenómenos colectivos, lo cual se ha demostrado con el análisis detallado de la teoría de las rutinas organizacionales en la TEE; otras se concentran exclusivamente en las características individuales de los componentes, como las teorías basadas en la psicología y en la estrategia que siguen los agentes en un contexto organizacional (véase por ejemplo la teoría del knowledge governance approach desarrollada por Foss, 2006). La propuesta principal es que ambas partes de la ecuación son indispensables para la construcción de una nueva teoría económica de la empresa, y que una nueva brecha de investigación se abre a partir de la interacción de estos dos aspectos, así como de la revisión crítica de las teorías existentes. 


\section{Bibliografía}

Antonelli, Cristino, Localized Technological Change. Towards the Economics of Complexity, Londres, Routledge, 2008.

Arrow, Kenneth, "The economic implications of learning by doing", en The Review of Economic Studies, vol. 29, núm. 3, junio de 1962, pp. 155-173.

Auyang, Sunny, Foundations of Complex-System Theories: In Economics, Evolutionary Biology, and Statistical Physics, Cambridge University Press, 1998.

Becker, Markus, "Organizational routines: a review of the literature", en Industrial and Corporate Change, vol. 13, núm. 4, 2004, pp. 643-677.

Becker, Markus, et al., "Applying organizational routines in understanding organizational change", en Industrial and Corporate Change, vol. 14, núm. 5, septiembre de 2005, pp. 775-791.

Coase, Ronald, "La naturaleza de la empresa”, en O. E. Williamson y S. G. Winter (comps.), La naturaleza de la empresa. Orígenes, evolución y desarrollo, México, FCE [1937], 1996 pp. 29-48.

Cohen, Wesley y Daniel A. Levinthal, "Innovation and learning: The two faces of R\&D", en The Economic Journal, vol. 99, núm. 397, septiembre de 1989, pp. 569-596.

Chandler, Alfred, The Visible Hand, Cambridge, MA, Harvard University Press, 1977.

Dosi, Giovanni, Marco Faillo y Luigi Marengo, "Organizational capabilities, patterns of knowledge accumulation and governance structures in business firms an introduction", Working Paper núm. 11, Pisa, Sant'Anna School of Advanced Studies, 2003.

Foss, Nicolai, "The emerging knowledge governance approach: challenges and characteristics", Working Paper núm. 06-10, Danish Research Unit for Industrial Dynamics, 2006.

Hodgson, Geoffrey, Economía institucional y evolutiva contemporánea, México, UAM, 2007.

Kitcher, Philip, El avance de la ciencia, México, Instituto de Investigaciones FilosóficasUNAM, 2001.

Koutsoyiannis, Anna, Microeconomía moderna, Buenos Aires, Amorrortu, 2002.

Levinthal, Daniel y James G. March, "The myopia of learning", en Strategic Management Journal, vol. 14, Special Issue: Organizations, Decision Making and Strategy, invierno de 1993, pp. 95-112.

Vol. 40, núm. 158, julio-septiembre / 2009
March, James, "Exploration and exploitation in organizational learning", en Organization Science, vol. 2, núm. 1, Special Issue: Organizational Learning: Papers in Honor of (and by) James G. March, 1991, pp.71-87.

Mantzavinos, Chrysostomos, Individuals, Institutions and Markets, Nueva York, Cambridge University Press, 2001

Nelson, Richard y Sidney Winter, "Evolutionary theorizing in economics", en The Journal of Economic Perspectives, vol. 16, núm. 2, primavera de 2002, pp. 23-46. , An Evolutionary Theory of Economic Change, Harvard, University Press, 1982.

"Neoclassical vs. Evolutionary theories of economic growth: critique and prospectus", en The Economic Journal, vol. 84, núm. 336, diciembre de 1974, pp. 886-905.

"Toward an Evolutionary Theory of economic capabilities", en The American Economic Review, vol. 63, núm. 2, mayo de 1973, pp. 440-449.

Patel, Pari y Keith Pavitt, "The technological competencies of the world's largest firms: complex and path-dependent, but not much variety", Research Policy, vol. 26, núm. 2, 1997, pp. 141-126.

Penrose, Edith, The Theory of the Growth of the Firm, Oxford, University Press [1959], 1995.

Prahalad, Coimbatore Krishnarao y Gary Hamel, "The core competence of the corporation", Harvard Business Review, vol. 68, núm. 3, mayo-junio de 1990, pp. 1-15.

Putterman, Louis y Randall S. Kroszner, The Economic Nature of the Firm, Nueva York, Cambridge University Press, 2006.

Schumpeter, Joseph, Capitalismo, socialismo y democracia, vol. I, Barcelona, Orbis [1942], 1983.

Valdaliso, Jesús María y Santiago López García, Historia económica de la empresa, Barcelona, Crítica, 2000.

Vromen, J., "Organizational routines and individual skills. Beyond analogy", Erasmus Institute for Philosophy and Economics Working Papers, enero de 2006.

Williamson, Oliver, Las instituciones económicas del capitalismo, México, FCE, 1989.

Winter, Sidney, "Coase, la competencia y la corporación", en Oliver Williamson y Winter Sidney (comps.), La naturaleza de la empresa. Orígenes, evolución y desarrollo, México, FCE, 1996, pp. 248-270.

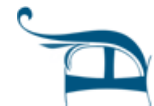

